\title{
MEPHISTO
}

\author{
The Board of the ICCA
}

For more than a year, there has been speculation concerning the future of Hegener + Glaser AG (Munich). During January 1994, any doubt still existing could be laid to rest when it became known that the company had been taken over by Saitek (Hong Kong). As we read it, the continuity of the Hegener + Glaser products has been ensured thereby.

The Board wishes to express their great appreciation to Hegener + Glaser for their substantial support given over many years to the ICCA in particular and to the chess community in general. Hegener + Glaser AG sponsored several ICCA Awards, computer-chess tournaments, and a number of "human" tournaments in which man and machine were able to participate on an equal footing. It is largely due to their efforts that, particularly in Germany, it is now commonplace to see the strongest commercially available chess computers taking part in tournaments with strong human masters and Grandmasters. The publicity so created contributes to human chess no less, we feel, than to computer chess.

Now that Hegener + Glaser AG have relinquished to some extent their corporate identity, this is the time to offer their founder members, Manfred Hegener and Florian Glaser, our sincere best wishes for their future endeavours.

Following this take-over, Saitek is now dominant in the chess-computer market. We have every reason to hope that, for the ICCA, the future will hold a successful collaboration with Saitek, a hope we base on their record of support of the ICCA in the past.

\section{THE BEST ANNOTATION AWARD}

\author{
The Editors
}

In the interests of stimulating wider use of chess-playing computers, the International Computer Chess Association has created a new prize to be awarded annually for the best computer-generated annotation of a chess game (cf. ICCA Journal, Vol. 15, No. 4, pp. 235-236). Each competing program will be given the same game (or the same short suite of games) to annotate. The program's output will be examined by a panel of independent experts (these will be internationally-known chess-players) who will assess the analysis based not only on the correctness and depth of the variations proposed, but also on the quality of the accompanying written comments. Authors have full freedom with their output, but it should be reminiscent of that appearing in newspaper chess columns. Authors are encouraged to include up to a maximum of three chess diagrams in their annotation of a game. Notation will be algebraic, with English letters as standard in this Journal or with figurines. Letters will stand for pieces as follows:

$\mathrm{P}=$ Pawn (optional), $\mathrm{Q}=$ Queen, $\mathrm{K}=$ King, $\mathrm{R}=$ Rook, $\mathrm{B}=$ Bishop, $\mathrm{N}=$ Knight.

The syntax of a move is:

$[<$ Piece $>$ ] $[<$ FromSquare $>]<$ ToSquare $>[=<$ PromotionPiece $>]$ where $<$ Piece $>$ is one of the letters $\mathrm{P}, \mathrm{Q}, \mathrm{K}$, $\mathrm{R}, \mathrm{B}$ or $\mathrm{N}$ and $<$ PromotionPiece> is one of $\mathrm{Q}, \mathrm{R}, \mathrm{B}$ or $\mathrm{N}$. The $<$ FromSquare $>$ and $<$ ToSquare $>$ are the normal algebraic coordinates of a square, for example, e 2 or $\mathrm{e} 4$.

To eliminate the possibility of operator interference, the programs should be capable of reading an ASCII file of a full game and should generate an output file that will be given to the review panel for examination. For input the programs will receive the full score of a game, in simple algebraic form, consisting of lines of input as follows:

$<$ DigitString $>$. $<$ WhiteMove $><$ BlackMove $>$

The following nonsense game illustrates castling on moves 5 and 7, en passant captures on moves 6 and 9 and capturing promotions on moves 10 and 11 . You are NOT asked to analyze this game, but it could serve as a test input. 